Check for updates

Cite this: J. Mater. Chem. B, 2018, 6, 7573

Received 24th August 2018 Accepted 17th October 2018

DOI: $10.1039 / c 8 t b 02239 a$

rsc.li/materials-b

\section{Seed-mediated synthesis of plasmonic gold nanoribbons using cancer cells for hyperthermia applications $\dagger$}

\author{
Ajay Vikram Singh, (D) *a Yunus Alapan, (D) ${ }^{a}$ Timotheus Jahnke, ${ }^{b}$ Peter Laux, (D) ${ }^{c}$

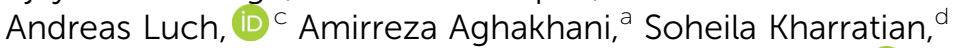 \\ Mehmet Cengiz Onbasli, ${ }^{e}$ Joachim Bill ${ }^{b}$ and Metin Sitti $\left(\mathbb{D}^{a}\right.$
}

A surfactant-less, seed mediated, biological synthesis of two dimensional (2-D) nanoribbons in the presence of breast cancer cells (MCF7) is demonstrated. The diameter and yield of nanoribbons are tunable via seeds and gold precursor concentration. Such crystalline nanoribbons serve to enhance the Raman signals over MCF7 cells. The side and slopes of the triangular nanoplatelets fused as nanoribbons exhibit plasmon excitement in quadrupole resonance modes in the infrared region. Consequently, when irradiated with an infrared laser they show an excellent photothermal effect and rapid rise in temperature. The experimental results verified by finitedifference time-domain (FTDT) calculations reveal the presence of wedge-plasmon polaritons propagating along the edges of the nanoribbons. These simulations confirm that long aspect ratio nanoribbon's edges and vertices act as an active optical waveguide, allowing for heat propagation along the long axis, killing cancer cells in the process at lower power doses.

The size, shape and surface properties of nanoparticles determine how they interact with biological materials. Varying these properties allows us to create novel approaches to develop nanoparticles for diverse therapeutic applications. ${ }^{1}$ Shape and size anisotropy of nanoparticles (NPs) critically influences their interactions with cells, such as in preventing phagocytosis, as well as their plasmonic properties, as applicable to photothermal therapy (PTT). ${ }^{2}$ As an example, rod-shaped gold nanoparticles exhibit superior plasmonic properties and enhanced cellular internalization when compared to spherical particles.

\footnotetext{
${ }^{a}$ Physical Intelligence Department, Max Planck Institute for Intelligent Systems, 70569 Stuttgart, Germany. E-mail: avsingh@is.mpg.de

${ }^{b}$ Institute for Materials Science, University of Stuttgart, Heisenbergstr. 3, 70569 Stuttgart, Germany

${ }^{c}$ Department of Chemical and Product Safety, German Federal Institute for Risk Assessment (BfR), Max-Dohrn-Strasse 8-10, 10589, Berlin, Germany

${ }^{d}$ Koc University, Department of Materials Science and Engineering, Sariyer, 34450 Istanbul, Turkey

${ }^{e}$ Koç University, Department of Electrical and Electronics Engineering, Sariyer, 34450 Istanbul, Turkey

$\dagger$ Electronic supplementary information (ESI) available. See DOI: 10.1039/c8tb02239a
}

The fabrication of anisotropic gold nanoparticles can be accomplished by a seed-mediated nucleation technique using a wet chemical method. ${ }^{3}$ However, this reaction requires toxic chemical reductants and stabilizers in solution, which limits its biomedical applications. ${ }^{4}$ Here, we present a biological route of anisotropic gold nanoparticle synthesis that is biocompatible and seeded on a bioorganic surface coating, known as a "biomolecular corona". 5 The shape of particles can be controlled during growth on the surface of cells using polypeptides that bind specifically to inorganic surfaces as functional molecules $^{6}$ or by involving membrane vesicles as templates for in situ engineering. ${ }^{7}$ However, a high-yield fabrication of high aspect ratio nanostructures, such as nanowires and nanoribbons, has not yet been demonstrated. ${ }^{8}$

Using the biological synthesis method, a small fraction of NPs with anisotropy has been produced by $E$. coli-secreted proteins containing specific polypeptide sequences, which induce the growth of flat triangular particles. ${ }^{9-11}$ Similarly, intra- and extracellular anisotropic NP growth in different cell lines are reported ${ }^{10}$ via a slow reduction process by varying culture conditions (serum vs. no-serum culture). ${ }^{6}$ However, the aqueous phase biological synthesis of nanoribbons under ambient conditions remains still unsolved. Such a benign route of synthesis, on one hand, makes nanoribbons highly biocompatible and on the other hand, their high aspect ratio would help for rapid propagation of photothermal plasmonic wave for biomedical applications. Herein, we present an in situ method of obtaining two-dimensional (2D) nanoribbons with a length of several micrometers grown on the surface of cancer cells. Our method also enables temporal control over the synthesis process by variation and tuning of the seed size, allowing for the versatile growth of different micro/nanostructures.

We have recently reported a biomineralization process, which turns ionic gold into spherical nanoparticles and anisotropic microplate structures, depending on the serum concentration of a cell culture medium. ${ }^{6}$ We were able to demonstrate that defense proteins related to cellular stress in cancer cells are able to reduce ionic gold and subsequently bind to specific crystal 
surfaces. This led to the anisotropic growth of gold NPs. Within $24 \mathrm{~h}$ of addition of the gold precursor solution, a sudden $\mathrm{pH}$ change of the cell culture medium occurred, the serum and cell membrane proteins in serum culture denatured, and the reduction of gold $\mathrm{Au}^{3+}$ to spherical nanoparticles as $\mathrm{Au}^{0}$ was triggered. The instantaneous $\mathrm{pH}$ drop led to an unfolding of the 3D protein structures, which enabled access to hydroxyl and thiol groups. Particularly, functional groups in tyrosine and tryptophan acted as electron donors and stabilizers for gold seeds and NPs, assisting in the reduction of $\mathrm{Au}^{3+}$ to metallic AuNPs ${ }^{12}$ as:

$$
\mathrm{AuCl}_{4}^{-}+3 \mathrm{e}^{-} \stackrel{\text { Tryptophan/Tyrosine }}{\longrightarrow} \mathrm{Au}_{(\mathrm{s})}+4 \mathrm{Cl}^{-}
$$

In a serum-free culture with $24 \mathrm{~h}$ of treatment, we did not observe any sign of anisotropic NP formation. However, after a long-term (up to a week) exposure, the spherical NPs acted as nucleation sites, where reduction of more $\mathrm{Au}^{3+}$ ions took place. With this method, using a slow reduction and seed-nucleated growth we have been able to fabricate triangular and hexagonal microplates. ${ }^{12}$ To further explore this mechanism, we hypothesized that this slow biological reduction method could be speeded up by adding cell-synthesized spherical gold NPs as a seed for anisotropic particle growth.

To test this hypothesis, we incubated mouse myoblast C2C12 and breast cancer cell line MCF7 cell monolayers with $1.5 \mathrm{mM} \mathrm{AuCl}_{4}$ salt solution under serum-free conditions. ${ }^{6}$ Eight hours after addition of the gold salt solution, we added $10 \mu \mathrm{L} \mathrm{mL}^{-1}$ of $20 \mathrm{~nm}$ spherical gold NPs, as seeds pre-synthesized by mammalian cells. Following a reaction time of $24 \mathrm{~h}$, we found worm-like structures on the culture surface as well as on the cell monolayer (Fig. 1A-F). Scanning electron microscopy (SEM) analysis demonstrated that the worm-like structures are triangular thin platelets fused tip-to-end, producing nanoribbon-like patterns in the process. Also, as qualitatively visible from the SEM images in
Fig. 1A-C compared to Fig. 1D-F, MCF7 cells fabricate more nanoribbons than $\mathrm{C} 2 \mathrm{C} 12$. Higher production might be related to the more versatile extracellular secretion of MCF7 cells compared to that of mouse myoblasts. ${ }^{13}$ We hypothesize that the nanoribbon synthesis under serum-free conditions is initiated by diffusion of gold ions from the bulk to the cell membrane via surface diffusion.

Over the cell membrane, reductions and irreversible incorporation of growth species (gold ions) into anisotropic thin microplatelet crystal structures take place via nucleation growth. ${ }^{6}$ When seed NPs are added, the anisotropic triangular platelet-like structures are fused together as long nanoribbons. From a thermodynamic perspective, a crystal lattice matching of the overlapping parts of the triangles occurs to minimize the interface energy. This is displayed in Fig. 2A schematic, showing the tip of one triangle overlapping another triangle along its side. Hereby the (111) lattice planes of both triangles match, resulting in the distinctive form of the nanoribbons. ${ }^{14}$ Further desorption and diffusion of the soldered chain of triangular particles take place from the surface to the culture medium, as shown via SEM images in Fig. 1A-F. The nanoribbon features are shown to mainly originate from protruding cellular filaments (e.g., filo/lammelipodia). We observed nanoribbons with a width of 50-200 nm (Fig. S2, ESI $\dagger$ ) and a length of up to $60 \mu \mathrm{m}$ protruding from the cell edges (Fig. S1A and B, $\mathrm{ESI} \dagger$ ). Among the different steps of the anisotropic growth, irreversible incorporation of growth species into the crystal structure, generally acted as the growth limiting step. In the presence of gold seeds and gold binding peptides (GBP) secreted under similar conditions, we expect preferential adsorption of GBP on the $\{100\}$ rather than $\{111\}$ crystal planes, which allows an ordered anisotropic growth to produce nanorods (NRs) and nanowires. However, we observed nanoribbons with high aspect ratios, which require a liquid crystalline phase with biomolecules from MCF7 cells as soft templates in bulk. ${ }^{15}$ In addition, the
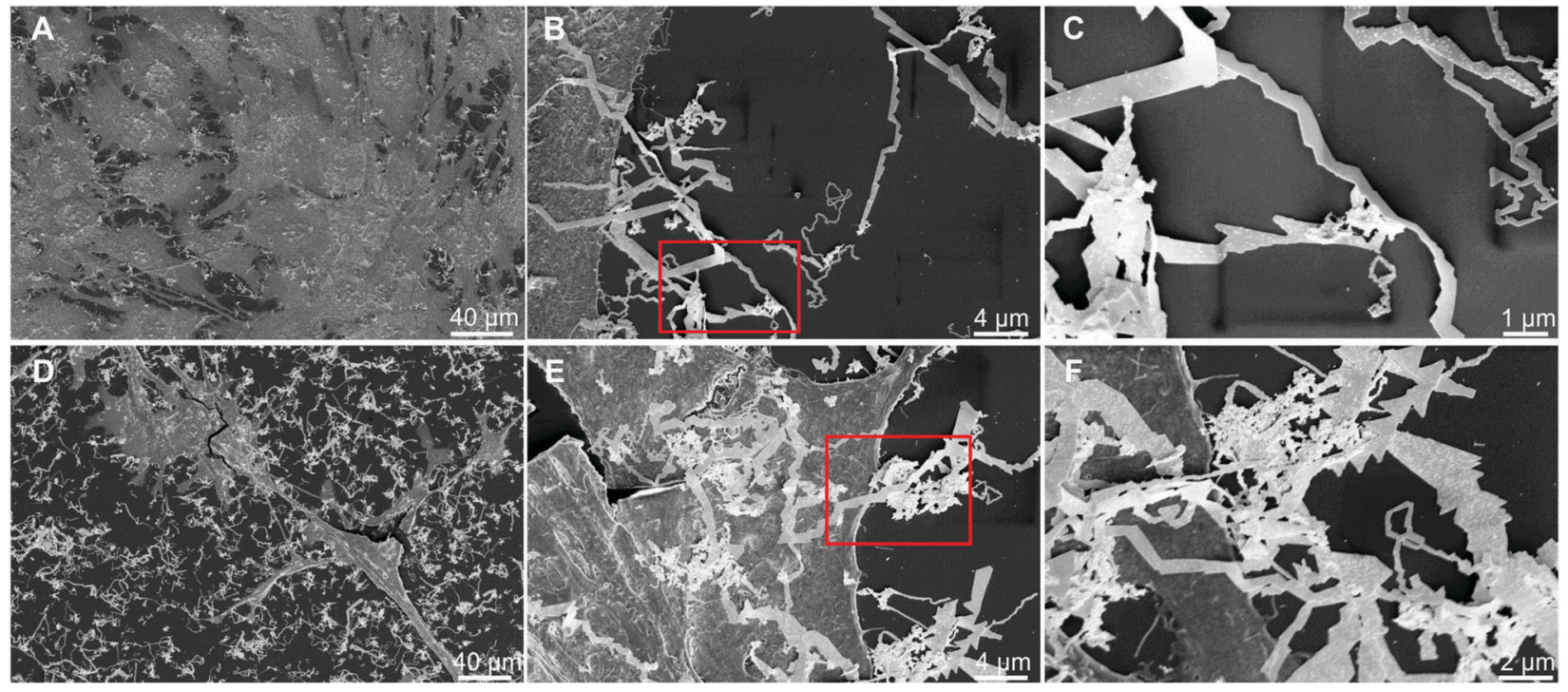

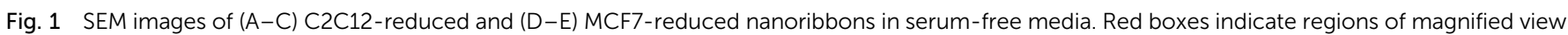
( $C$ and $F$ ) of attached gold nanoribbons on the cell membrane formed via nucleation-mediated growth of anisotropic structures. 

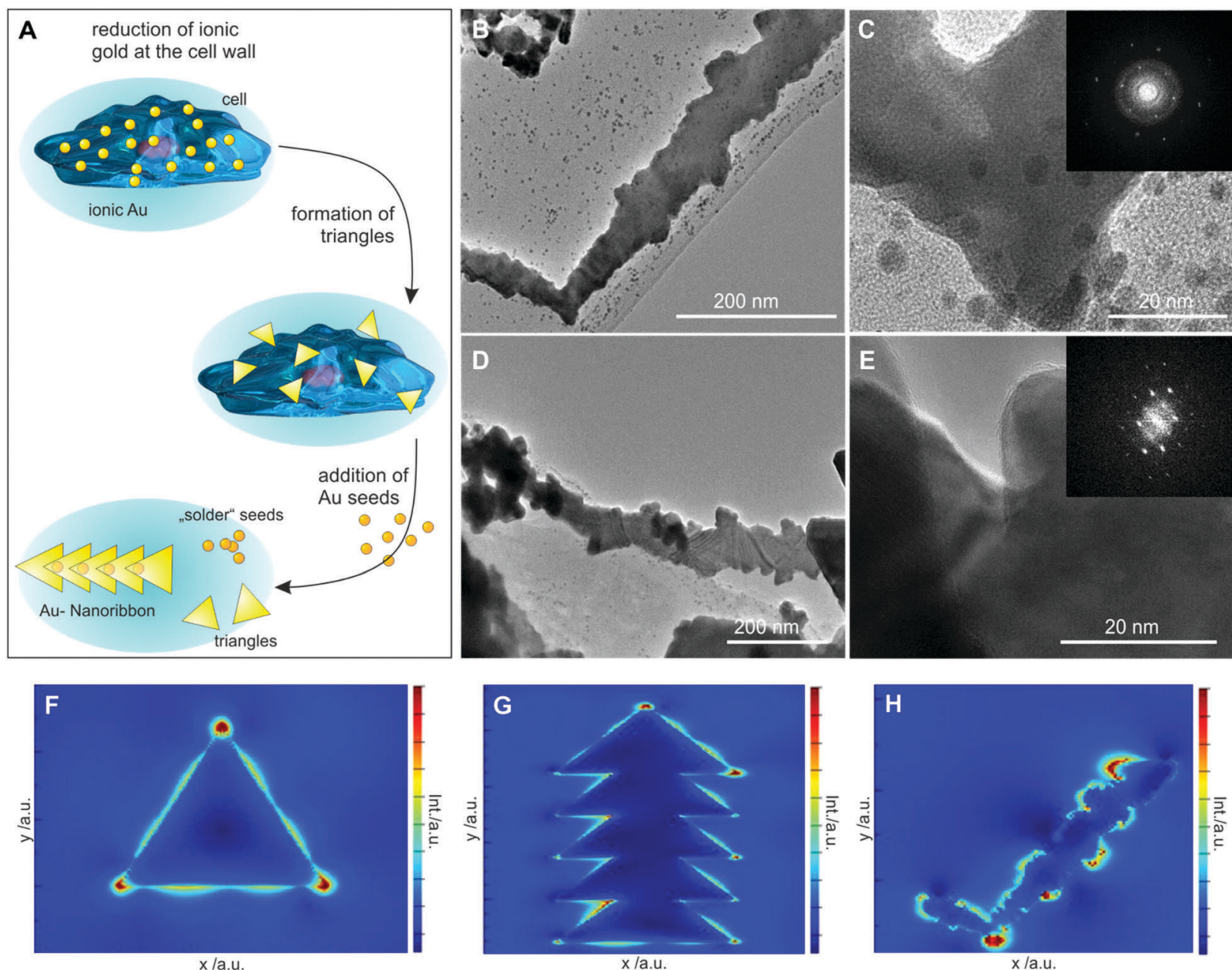

Fig. 2 (A) Schematic showing the different steps of the biological synthesis of nanoribbons. TEM analysis in low (B) and high (C) magnifications shows soldering with spherical NP seeds (inset: diffraction pattern along the [111] direction). (D) TEM analysis of the edge of the sheets shows the rough, crystalline nature of the edges in the high-magnification TEM image (E). Inset images show the selected area electron diffraction from the samples $(C$ and $E)$, respectively. $(F-H)$ simulated mode profiles of the surface plasmon resonances (SPR) of a single triangular nanoplate (F), fused platelets as a train of nanotriangles $(G)$ and TEM images shown in $B(H)$.

secreted GBP must have several times more hydrophobicity with better interfacial adsorption to promote nanoribbon growth in the presence of NP seeds. ${ }^{16}$

Compared to the recent work showing multiply-twinned platelet and hexagon synthesis using cancer cells, herein, fused thin nanoplate synthesis with a twinned layer in the range of 150-200 nm could have a vital influence for biomedicine applications, since the thickness of the nanoplates affects the plasmon resonance. ${ }^{17}$ Contrary to thin platelets, end-to-end arranged multiply-twinned nanoribbon structures exhibit very diverse plasmon transmission through the corners and propagation along the edges. ${ }^{18}$ These unique features could contribute to the enhanced photothermal destruction of tumors via utilization of these nanoribbons as long-range plasmonic waveguides. Further experiments were carried out using only the cancerous MCF7 cells to demonstrate cancer cell killing. We next investigated the influence of seed and gold ion concentration on nanoribbon size and shape control. Increasing the gold ion concentration from $1.5 \mathrm{mM}$ to $2 \mathrm{mM}$, while keeping the seed concentration the same, resulted in a change of nanoparticle shapes from nanoribbons to triangular and hexagonal microplates (Fig. S3A and B, ESI $\dagger$ ). In contrast, varying the concentration of seed NPs from $10 \mu \mathrm{L} \mathrm{mL} L^{-1}$ to $25 \mu \mathrm{L} \mathrm{mL}^{-1}$ with $1.5 \mathrm{mM}$ gold ions, an increase in the yield of nanoribbons was observed (Fig. S3C and D, ESI $\dagger$ ). High-resolution transmission electron microscopy (HR-TEM) analysis demonstrated that these nanoribbons are formed by thin, single crystalline flake-like units joined together at regular intervals (Fig. 2B-E). Seed particles, added to accelerate the reaction, did not only help in seedmediated nucleation and growth but also played a crucial role in fusing triangles into nanoribbons.

As shown in TEM images in Fig. 2B, quasi-L shaped nanoribbons show multiple spherical seed nanoparticles near the bend of L-shaped ribbons, indicating that these units are independently formed via seed-mediated reduction. The band-like patterns shown in a single crystal triangle arise due to the difference in the electron density caused by bending or deformation of the nanoribbon possibly as a consequence of the 
radiation effect of the electron beam as reported in thin metal foils (Fig. 2D-E). ${ }^{8}$ TEM analysis of the edge of the sheets (Fig. 2E) shows the rough nature of the edges of the gold prisms, indicating a biological corona formed at the edges. Particle attachment seems to occur in different stages in the scheme (Fig. 2A): the seed particles attach to the triangles, preferably at the edges of the sheets, in order to reduce the surface energy. Then the seed particles match the underlying crystal structure of the gold triangles. These gold NPs seem to be in a liquid gold state and align to the single crystalline prism. ${ }^{19,20}$ Upon coming into contact with another triangle, the aligned seed particles act as a glue between them and an oriented attachment occurs between both. Repetition of this process leads to long shapes, composed of triangles, which are overlapping tip-to-side, in order to match their crystal lattice structure on the (111) facet. This correlates well with the HR-TEM image in Fig. 2E, showing a nearly perfect single crystal structure. The interplanar lattice distances could be measured as $0.247 \mathrm{~nm}$. The electron diffraction pattern exhibits a crystal texture in the (111) direction, which is caused by the large (111) facet facing upward and being perpendicular to the beam axis.

The growth of nanoribbons by an intermediate liquid gold is in agreement with our recent report; ${ }^{20}$ however, further work is warranted. We measured surface-plasmon resonances of individual thin triangular gold platelets and their transformed nanoribbon structures using 3D finite difference time domain (FDTD) simulations at high spatial resolution. The simulation results demonstrate the presence of wedge-plasmon polaritons propagating along the nanoplatelets and nanoribbons (Fig. 2F-H). Extracting the surface features from the TEM and SEM images and directly applying the FDTD simulation clearly demonstrate that the edges of nanoribbons act as plasmonic waveguides for rapid transmission of optical signals.

Energy dispersive X-ray (EDX) analysis confirms the presence of gold and carbon/nitrogen/oxygen arising from the proteinaceous/biomolecular organic phase (Fig. S3E, F and S4A-E, ESI $\dagger$ ). X-ray photoelectron spectroscopy (XPS) analysis of the nanoribbons has further confirmed the presence of metallic gold with a small fraction of $\mathrm{Au}(\mathrm{I})$ gold (Table 1) still present. The binding energies of $\mathrm{Au}$ if doublets with a peak at $84.4 \mathrm{eV}$ and $87.8 \mathrm{eV}$ correspond to $\mathrm{Au} 4 \mathrm{f}_{7 / 2}$ and $\mathrm{Au} 4 \mathrm{f}_{5 / 2}$, respectively. They are caused by the metallic nature of nanoribbons.

An additional peak was observed at $90.8 \mathrm{eV}$ representing the $\mathrm{Au}(\mathrm{I})$ state, which is related to partially reduced ionic gold within the nanoribbon network. We further noted amide-, carbonyl- and alkyl-associated carbon peaks, which arise due to electron emissions from the diverse organic material associated with these nanoribbons (Fig. 3A-C). The XPS N 1s spectra were assigned to amine/amide (400.5 eV) from the nanoribbon

Table 1 XPS deconvoluted peak positions of gold

\begin{tabular}{lllcr}
\hline Name & Position & FWHM & Height CPS & \multicolumn{1}{c}{ Area } \\
\hline $4 \mathrm{f}_{5 / 2}$ & 87.8 & 1.46 & 645.75 & 1043.72 \\
$4 \mathrm{f}_{5 / 2}$ & 90.8 & 1.46 & 291.25 & 470.74 \\
$4 \mathrm{f}_{7 / 2}$ & 84.5 & 1.46 & 1192.97 & 1928.21
\end{tabular}

surface indicating the role of secreted proteins in the synthesis process. $^{21}$

Rapid analysis of the biochemical composition of a single living cell using non-invasive, highly sensitive, and label-free techniques has important implications in cell-based micro- and nanotechnologies for biomedical applications. Surface-enhanced Raman scattering (SERS) provides a non-destructive solution for such single cell analysis methods at an unprecedented resolution. ${ }^{22}$ Raman spectra recorded at $632 \mathrm{~nm}$ incident wavelength from control cells without gold ion treatment were noisy, and individual Raman peaks were not discernible (compare Fig. 3D-F and G-I). However, gold ion-treated cells showed a series of bands that corresponded to diverse biopolymers also found in the cells (Fig. 3G-I). We observed the nucleic acid peaks at $1067 \mathrm{~nm}\left(\mathrm{PO}_{2}\right.$ in DNA) and $831 \mathrm{~nm}\left(\mathrm{PO}_{2}\right.$ in RNA), Protein Amide(III) at $1220 \mathrm{~nm}$ and amino acids phenylalanine at $1000 \mathrm{~nm}$, tyrosine at $854.1 \mathrm{~nm}$, and tryptophan at $754 \mathrm{~nm}$. The lipid peaks between 1448-1460 nm display shoulders which might arise from the direct interaction with the nanoribbon surface, as lipids are known to stabilize and modify the NP surface corona. ${ }^{23}$ Sugar ring associated $\mathrm{C}-\mathrm{O}-\mathrm{C}$ Raman peaks were identified spreading between $800-1500 \mathrm{~nm} .{ }^{24}$ The characteristic peaks of fixed cells around $1005 \mathrm{~nm}$ were found diminished due to enhanced signals associated with nanoribbons. ${ }^{22}$ Mono-substituted aromatic compounds exhibit strong ring vibration at $1000 \mathrm{~nm}$ (e.g., Phe, Trp) and $850 \mathrm{~nm}$ (e.g., Tyr), with a slight shift arising due to different substitution symmetry as observed in our analyses..$^{25}$ The presence of these amino acids further corroborates our hypothesis of protein-mediated reduction/capping and seed-nucleation growth of nanoribbons reported herein. ${ }^{26}$

Metallic materials are of great interest for near-infrared (NIR) hyperthermia applications since they show great promise as efficient tools for tumor ablation. ${ }^{27}$ Gold nanoparticles, nanorods, and nanowires have been reported as potential materials; yet clinicians are in quest of superior hyperthermia agents, which are easy to synthesize, incorporate and give efficacious results. ${ }^{4,27}$ Promising results have been obtained from previous reports of hybrid nanowires of gold NPs decorated with silica; however, they require PEGylation for higher uptake and relatively higher laser power to destroy cancer cells. ${ }^{28}$ Comparatively, cell-based, seed-mediated growth of nanoribbons described in this report has several advantages. First, since biological cells synthesize the nanoribbons, they are highly biocompatible and no functionalization is required for cellular uptake. Second, lower laser power and radiation dose are needed for tumor ablation due to a sharp rise in temperature upon laser irradiation (Fig. S6, ESI $\dagger$ ). Finally, strong NIR absorption is observed due to plasmon excitement via the edges for trigonal platelets in the $100-200 \mathrm{~nm}$ thickness range ${ }^{18}$ (UV-vis spectra in Fig. S7, ESI $\dagger$ ). The side and slopes of the triangular nanoplatelet-ribbons exhibit plasmon excitement in quadrupole resonance modes involving complex field distribution on the surfaces in the infrared region. Therefore these nanoribbons could be powerful NIR hyperthermia agents, complementing photothermal tumor ablation, rivaling the state-of-the-art cancer hyperthermia agents reported recently. 

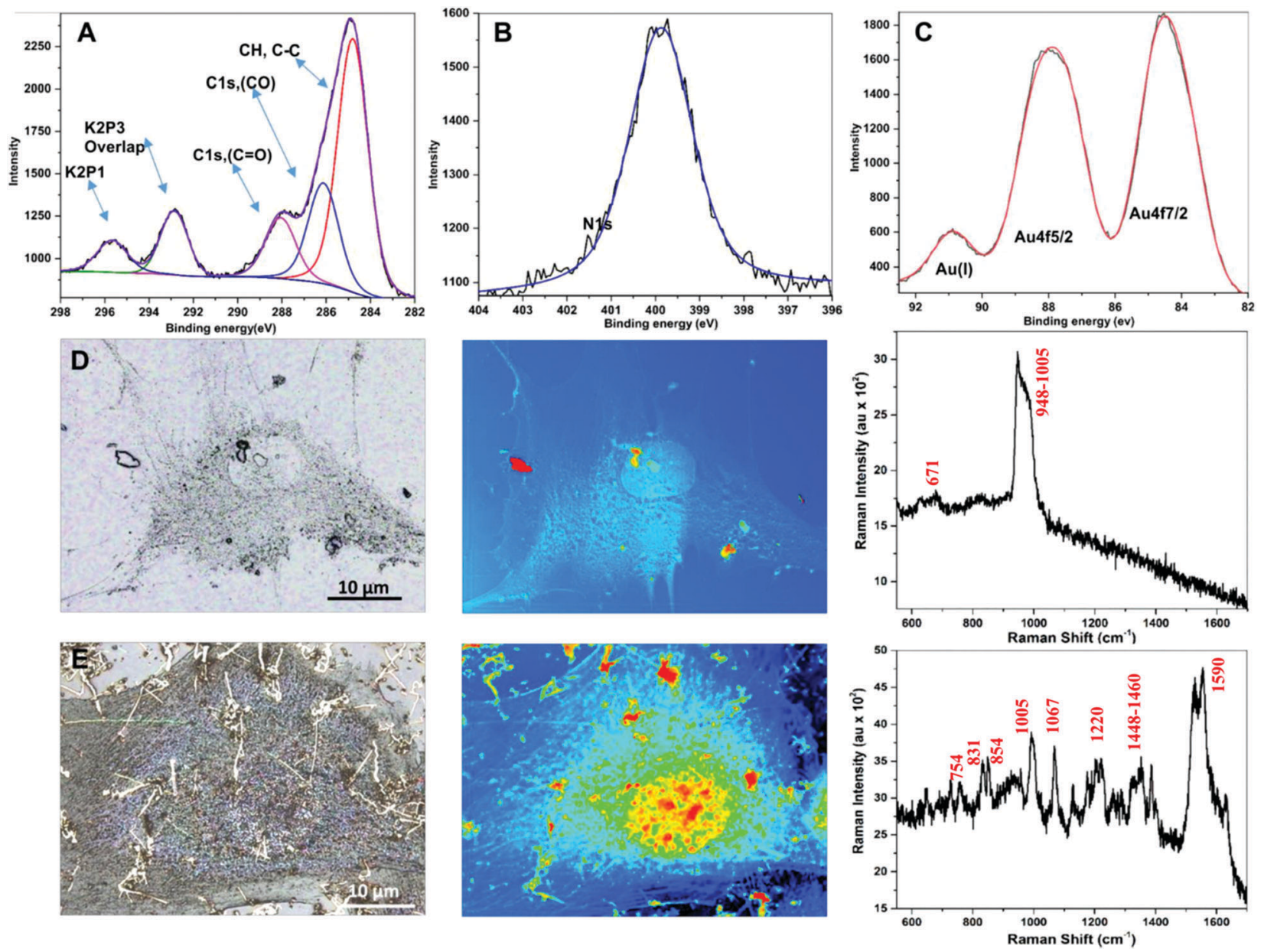

Fig. 3 High-resolution XPS spectra showing (A) carbon (C 1s), (B) nitrogen (N 1s), and (C) gold 4f7/4f5 nanoribbon-associated orbital binding energies of MCF7 cells in serum-free culture medium. Typical optical ( $D$ and G), SERS map images ( $E$ and $H$ ) and mean SERS spectrum (F and I) of twelve spectra at different points inside the MCF7 cell nucleus without $(D-F)$ and with gold $(G-I)$ nanoribbon synthesis.

To test the photothermal efficacy, the nanoribbons obtained in aqueous solution were purified overnight inside a dialysis bag with a $340 \mathrm{kDa}$ molecular weight cut-off. Subsequently, nanoribbons were irradiated with a $780 \mathrm{~nm}$ NIR laser at a power density of $\sim 0.6 \mathrm{~W} \mathrm{~cm}^{-2}$ inside Dulbecco's Modified Eagle Medium (DMEM), water and phosphate buffered saline (PBS) as controls. Compared to the control group, where we found no obvious increase in temperature, nanoribbons exhibited a significant increase in temperature when exposed to the NIR laser. Compared to the temperature change obtained with commercial nanorods used in photothermal therapy and spherical nanoparticles synthesized with breast cancer cells reported in our previous protocol, nanoribbons demonstrated a superior photothermal effect upon irradiation with the NIR laser. ${ }^{6,29}$ Maximum temperatures recorded after NIR laser irradiation correlates with these findings: gold nanoribbon $\left(65.5{ }^{\circ} \mathrm{C}\right)>$ gold nanorods $\left(50.2{ }^{\circ} \mathrm{C}\right)>$ spherical NPs $\left(39 .{ }^{\circ} \mathrm{C}\right)($ Fig. $4 \mathrm{~A}-\mathrm{C})$. We believe that tip-to-side soldered thin nanoplatelets in nanoribbons act as photothermal transducers or antennas to absorb the light. ${ }^{30}$ The large anisotropy in shape significantly enhances the conversion of external light to thermal heat. Furthermore, they even convert the energy more efficiently than anisotropic gold nanorods/spherical NPs. $^{29,30}$

Additionally, we evaluated the photothermal ablation capacity of the nanoribbons to investigate in vitro hyperthermia of breast cancer cells MCF7. For this, we incubated MCF7 cells with gold ions to synthesize gold nanoribbons, as described in the previous section, and spherical NPs in situ, as reported recently. ${ }^{6}$ Subsequently, cells were exposed to a $780 \mathrm{~nm}$ laser of $\sim 0.6 \mathrm{~W} \mathrm{~cm}^{-2}$ for $5 \mathrm{~min}$ at three different spots. In a parallel control experiment, we incubated MCF7 cells with PEGylated gold nanorods before NIR irradiation. After NIR irradiation, we stained the cells with trypan blue and Calcein as shown Fig. 4D-G and H-K, respectively. The breast cancer cells with nanoribbons showed the largest number of dead cells (lowest cell viability) compared to controls (untreated cells), spherical gold NPs, and gold nanorods after NIR ablation (Fig. S8, ESI $\dagger$ ). These in vitro hyperthermia experiments demonstrate that gold nanoribbons could be highly efficacious for in vivo tumor ablation as compared to other NIR sensitive anisotropic gold nanostructures reported so far.

In conclusion, we have reported the first example of the seed-mediated in vitro biological synthesis of nanoribbons on 

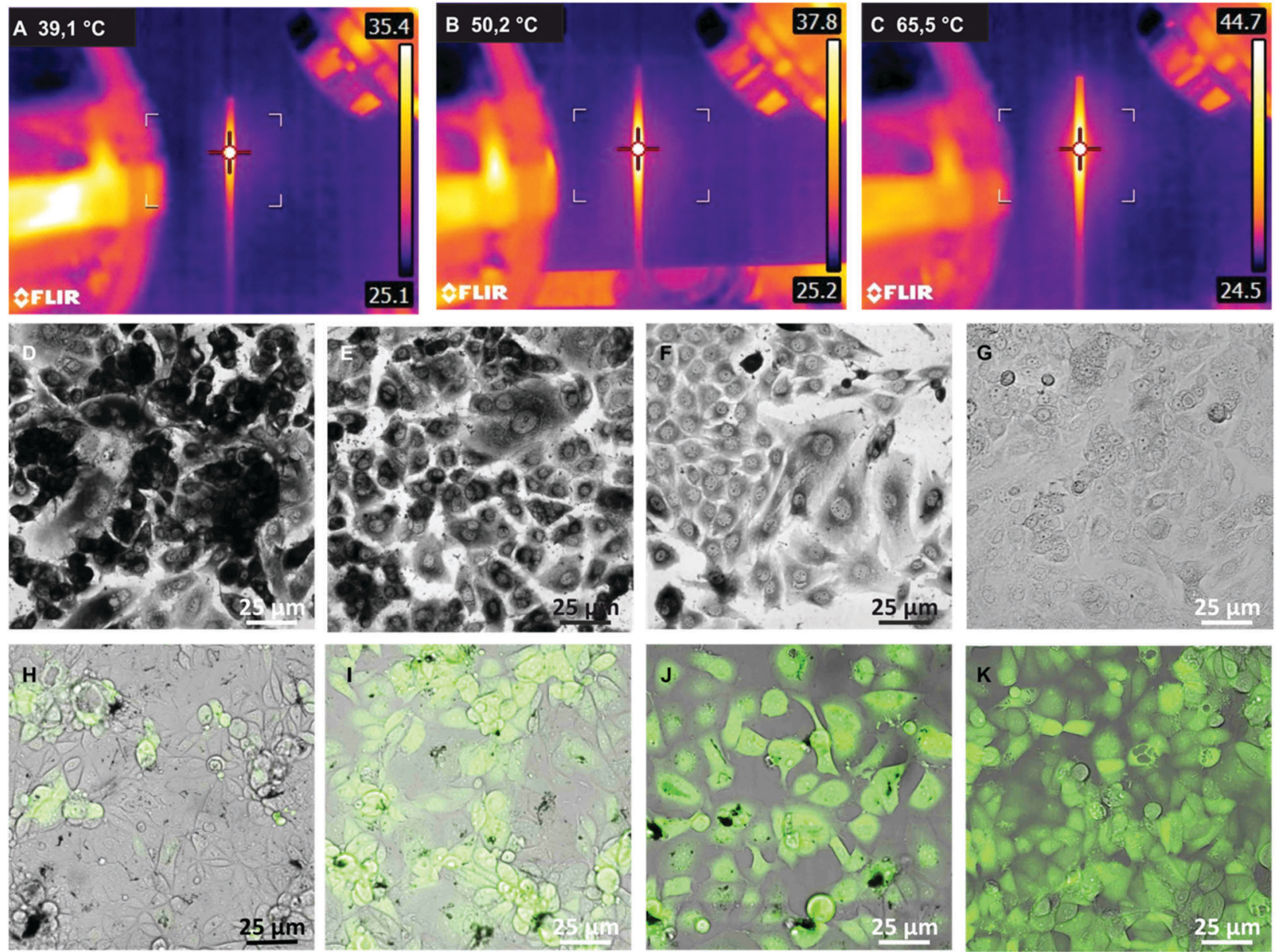

Fig. 4 Thermograms after laser power deposition to microcapillaries filled with spherical gold NPs as controls (A), PEGylated gold nanorods (B) and nanoribbons (C) as controls using our protocols. Optical images of MCF7 cells with trypan blue-stained dead cells (middle panel) and Calcein AM stained live cells (lower panel). Optical (D) and overlay of fluorescent-phase contrast microscopic images. (H) Nanoribbons synthesized from MCF7 cells $\left(50 \mu \mathrm{g} \mathrm{mL}{ }^{-1}\right.$ Au nanoribbons), (E and I) PEGylated-Au nanorod $\left(50 \mu \mathrm{g} \mathrm{mL}^{-1}\right.$ ) treated MCF7 cells, (F and J) spherical gold NP (50 $\mu \mathrm{g} \mathrm{mL}{ }^{-1}$ ) treated $\mathrm{MCF}$ cells, and $(G-K)$ control MCF7 cells without any treatment after NIR laser irradiation.

the surface of cancer cells. The one-pot, in situ methods reported herein show batch production of the nanoribbons with high reproducibility. The nanoribbons could be used as powerful SERS imaging and NIR hyperthermia agents, complementing photothermal tumor ablation and rivaling the state-of-the-art cancer hyperthermia agents reported recently. The present in vitro findings suggest new opportunities for novel gold-based cancer nanomedicine. Nonetheless, further work is necessary for a better understanding of the in vivo efficacy of cancer killing of these nanoribbons within a tumor micro-environment. The effect of aspect ratio on photothermal conversion is also a matter of further investigation in lieu of novel emerging biomedical applications for hyperthermia, particularly in bacterial biofilm treatment.

\section{Experimental section}

\section{Materials}

Chloroauric acid $\left(\mathrm{HAuCl}_{4}\right)$ was purchased from Sigma Aldrich. The cell counting Kit-8 (cat \#CK04-20) was purchased from Tebu-bio,
Offenbach, Germany. PEGylated gold nanorods were purchased from Nanopartz Inc. CO, USA. Cell culture materials (FBS, DMEM, Penicillin/streptomycin, Glutamate) were purchased from Gibco, Germany. Calcein AM and trypan blue were purchased from VWR chemicals, Germany. All chemicals and reagents were used without additional purification. Except where described otherwise, all solutions were prepared using Milli-Q water (Millipore $\mathrm{GmbH}$ Germany) as the solvent. Optical measurements were performed at room temperature under ambient air conditions. UV-vis absorption spectra were recorded with a Synergy UV-vis-near-infrared (BIOTEK, USA) spectrophotometer with ZEN 5 analysis software.

\section{Seed-mediated synthesis of nanoribbons with cell lines}

Human breast adenocarcinoma MCF7 and C2C12 mouse myoblast cell lines were used as model mammalian cells to investigate nanoribbon synthesis under serum-free conditions. Filter sterilized $1.5 \mathrm{mM} \mathrm{HAuCl}{ }_{4}$ solution (pore size $0.22 \mu \mathrm{M}$ ) in phosphate buffer saline (PBS), $\mathrm{pH}$ 7.4, was used as a standard molar concentration for different characterization. After the cells reached confluency, sterile $1.5 \mathrm{mM} \mathrm{HAuCl}_{4}$ was directly 
added to Dulbecco's Modified Eagle Medium (DMEM) supplemented with $10 \%$ fetal bovine serum (FBS). Under serum-free conditions, the growth medium of the confluent monolayer of cells was replaced with $1.5 \mathrm{mM} \mathrm{HAuCl}{ }_{4}$ supplemented sterile PBS. After 8 hours, $10 \mu \mathrm{L} \mathrm{mL}{ }^{-1}$ presynthesized spherical gold NPs with MCF7 cells were added and the culture was incubated at $37{ }^{\circ} \mathrm{C}$ and in $5 \% \mathrm{CO}_{2}$ atmosphere in a sterile incubator within a T25 culture flask. The appearance of a brownish or blackish color on the cell surface due to anisotropic nanoribbons after 24-36 hours was indicative of the formation of nanoribbons. No color change was observed in the negative controls: dead cells incubated with $\mathrm{PBS}$ and $\mathrm{HAuCl}_{4}$; live cells incubated with PBS and without $\mathrm{HAuCl}_{4}$; PBS incubated with $\mathrm{HAuCl}_{4}$ without cells. In PBS, nanoribbon solutions are stable for months, indicating that autoreduction does not occur.

\section{Cell proliferation and cytotoxicity assay}

MCF-7 was seeded in Ibidi ${ }^{\circledR} 8$ chambered microwells with glass bottom at a seeding density of $5 \times 10^{4}$ cells $\mathrm{mL}^{-1}$ in complete low glucose DMEM (Life Technologies, Germany) and grown for 3 days (70\% confluency, $\sim 10^{6}$ cells per well) at $37{ }^{\circ} \mathrm{C}$ in a humidified incubator containing $5 \% \mathrm{CO}_{2}$. An overnight culture of MCF-7 was established in a 96 well plate. HAuCl4 solution in sterile PBS was directly added to the MCF-7 confluent monolayer. After 48 hours of incubation, the cells were washed thoroughly with sterile PBS $(1 \times)$ to wash away unreacted gold ions or free-floating nanoribbons. Biocompatibility was assayed with the cell counting Kit-8 (cat \#CK04-20, Tebu-bio), which is a colorimetric assay for the determination of viable cell numbers and can be used for cell proliferation assays as well as cytotoxicity assays. The cell counting Kit-8 uses a tetrazolium salt, WST-8, which produces the water-soluble WST-8 formazan. Since this orange colored formazan does not require dissolving, no solubilization process is required. Results are obtained after three simple steps: by (1) adding $10 \mu \mathrm{L}$ of CCK-8 solution to each well of the 96 plate, (2) incubating the plate for 1-4 hours in the incubator and (3) measuring the absorbance at $450 \mathrm{~nm}$ using a microplate reader. WST-8 is not cell permeable, which results in low cytotoxicity.

\section{NIR-triggered hyperthermia comparison between commercial gold nanorods, spherical nanoparticles and nanoribbons synthesized from cancer cells}

The photothermal properties of anisotropic gold nanoribbon structures and spherical nanoparticles synthesized from cells, as well as commercial gold nanorods, were tested. Samples were loaded in capillary tubes ( $50 \mu \mathrm{g}$ Au per $\mathrm{mL}$ ) as determined with inductively coupled plasma mass spectrometry (ICP-MS). Tubes containing $200 \mu \mathrm{L}$ of samples were irradiated under NIR $\left(780 \mathrm{~nm}, \sim 0.6 \mathrm{~W} \mathrm{~cm}^{-2}\right.$ ). Thermal images were collected using an infrared thermal camera (ETS320, Flir Systems, Wilsonville, OR, United States). For in vitro hyperthermia demonstration, breast cancer cells (MCF7, ATCC/DSMZ number: HTB-22, Passage 8) were incubated with gold ions, for the synthesis of gold nanoribbons and spherical nanoparticles, and PEGylated gold nanorods, as a control. To quantify cell viability, cells were irradiated with a NIR laser of $780 \mathrm{~nm}$ and $\sim 0.6 \mathrm{~W} \mathrm{~cm}^{-2}$ for $5 \mathrm{~min}$ at three different spots. Following irradiation, cells were imaged using phase and fluorescence microscopy after staining with Calcein (30 minute incubation at $5 \mu \mathrm{g} \mathrm{mL}{ }^{-1}$ in culture medium; Invitrogen), a fluorescent indicator of esterase activity in viable cells.

\section{Trypan blue assay}

Cells were cultured in a $35 \mathrm{~mm}$ dish overnight. The medium was removed, and solutions of purified nanoribbons in PBS $\left(50 \mu \mathrm{g} \mathrm{mL} \mathrm{m}^{-1}, 2 \mathrm{~mL}\right.$ ) were added to the dish, followed by $5 \mathrm{~min}$ NIR laser irradiation at a power density of $0.6 \mathrm{~W} \mathrm{~cm} \mathrm{~cm}^{-2}$. The suspensions were removed and the $0.4 \%$ trypan blue were added to cells for $10 \mathrm{~min}$. The trypan blue was removed and the cells were washed with PBS three times, and bright-field microscopy was carried out.

\section{X-ray photoelectron spectroscopy}

XPS analysis was performed with a Thermo VG Thetaprobe 300 (Thermo Fischer Scientific) system using monochromatic incident $\mathrm{Al} \mathrm{K} \alpha$ radiation $(h \nu=1486.68 \mathrm{eV}$; spot size $400 \mu \mathrm{m}$; base pressure $<10-7 \mathrm{~Pa}$; average detection angle of $53^{\circ}$ with respect to the sample surface). Energy calibration, removal of contaminants, and charge compensation during the measurements were carried out. The XPS spectra were deconvoluted with the Thermo VG Scientific Advantage 5.47 software (Thermo Fischer Scientific). Samples were prepared by fixing the cells, dehydrating in graded ethanol (1\%-25\%-50\%-70\%-100\%) and air drying. Nanoparticles and microplates suspended in DI water were drop coated onto silicon wafer chips $(5 \times 6 \mathrm{~mm})$ and air-dried overnight.

\section{FTIR analysis}

Chemical imaging of cells with and without $\mathrm{HAuCl}_{4}$ was done using a Bruker Tensor II spectrometer with an ATR sensor. Cells were grown to $100 \%$ confluence on a flat glass substrate and treated with gold ions and incubated for 4 days. Subsequently, medium contaminants were removed and cells were washed with PBS and fixed in formalin. Cells were washed in HBSS before IR analysis after formalin fixations to remove residual phosphate ions. The sample was placed over the ATR-crystal carefully and pressed with a platinum diamond ATR-accessory to avoid uneven thickness and make close contact of the sample with the ATR crystal. IR spectra was recorded in absorbance mode with $4 \mathrm{~cm}^{-1}$ resolution and 1024 scans. Data analysis was performed with OPUS.

\section{DLS and zeta potential measurement}

The hydrodynamic diameter of the nanoparticles was determined using a light scattering instrument (MÖBIU $\zeta^{\circledR}$ analyzer, Wyatt technology Germany) with an ATLAS pressurization system. The zeta potential was calculated from measurements of electrophoretic mobility performed by the same equipment.

\section{Scanning electron microscopy imaging}

A Zeiss Ultra 55 Gemini scanning electron microscope was used for imaging MCF7 cells with nanoparticles/microplates. 
An accelerating voltage of $3 \mathrm{keV}$ and a SE detector were used for imaging. To prepare samples for SEM, cells were incubated with $1 \mathrm{mM} \mathrm{HAuCl} 4$ for 4 days. After the incubation period, cells were fixed in $2.5 \%$ glutaraldehyde in PBS for $45 \mathrm{~min}$ at $4{ }^{\circ} \mathrm{C}$, rinsed with PBS, then water. Cells were dehydrated in a series of graded aqueous ethanol concentrations $(30 \%, 50 \%, 70 \%, 90 \%$, and $100 \%$ ) for 5 minutes in each solution and 10 minutes in pure ethanol. Cells were further dehydrated and preserved using an automated critical point dryer (Leica EM, CPD 300). Wafers with cells were air dried followed by sputtering deposition of $5 \mathrm{~nm}$ nickel using a Leica coating system (Leica EM, ACE600).

\section{Transmission electron microscopy analysis}

In situ TEM observation of gold nanostructures in the intra- and extracellular environment of MCF7 cells was performed using a JEM-2100HR transmission electron microscope (JEOL, Japan) operated at $100 \mathrm{kV}$ equipped with an energy-dispersive X-ray (EDX) attachment. The cells were harvested by a cell scraper and suspended in PBS. Then the cells were washed three times by centrifugation (2000 rpm, $5 \mathrm{~min})$. Precipitates were fixed in $3 \%$ glutaraldehyde in HEPES buffer ( $\mathrm{pH} 7.4$ ) for $2 \mathrm{~h}$. After dehydration, the cells were embedded in Epoxy 618. Ultrathin sections of the MCF7 cells were acquired using an EM-UC6 microtome (Leica Co., Austria). Cells were stained with uranyl acetate and lead citrate prior to examination with TEM. Nanoparticles and microplates in the extracellular medium were drop coated on suspended monolayer graphene on TEM grid substrates (Quantifoil gold, Sigma) for high-resolution imaging and electron diffraction.

\section{Finite difference time domain simulations}

The 3D finite difference time domain (FDTD) simulations were performed using Maxwell's solver of Lumerical software. The absorbing boundary conditions were assumed for FDTD solutions. Meshing was composed of $5 \mathrm{~nm}$ cubes for a single gold triangle and chain of gold triangles (Fig. $2 \mathrm{~F}-\mathrm{H}$ and Fig. S5, ESI $\dagger$ ), and $7 \mathrm{~nm}$ cubes for the reconstructed SEM/TEM image of gold nanoribbons (Fig. 2F-H and Fig. S5, ESI $\dagger$ ). A circularly polarized plane wave at $\lambda=780 \mathrm{~nm}$ was used for excitation of the samples. Optical properties of the gold NPs were taken from Palik's Handbook (Handbook of Optical Constants of Solids). Polystyrene (refractive index of 1.587 and extinction coefficient of 0 at $632.8 \mathrm{~nm}$ ) and PBS (refractive index of 1.3325 and extinction coefficient of $7.2792 \times 10^{-9}$ ) were chosen as the substrate and medium, respectively. For extracting the features from the SEM images, an image processing with a specific threshold was applied to get a clear binary picture of the gold nanoribbons and then imported it to Lumerical software as a surface and finally extruded it to the desired thickness.

\section{SERS microscopy}

The NanoRaman ${ }^{\mathrm{TM}}$ platform integrates an inverted optical microscope and near-field optical techniques (SNOM or NSOM) (HORIBA Scientific Germany). An instrument equipped with a liquid nitrogen-cooled CCD detector was used to evaluate the SERS imaging of control and MCF7 cells. The SERS mapping and SERS spectra were recorded, with $100 \mu \mathrm{m} \times 100 \mu \mathrm{m} \times 6 \mu \mathrm{m}$ scan-range in the $X Y Z$ directions with $200 \mathrm{~nm}$ resolution in the $X-Y$ plane and $500 \mathrm{~nm}$ resolution in the $Z$-plane respectively. SERS spectra, SERS mapping and confocal images were recorded using a NIR laser emitting at $780 \mathrm{~nm}$ with an irradiation laser power of $3 \mathrm{~mW}$ on the sample plane. Twelve scans of $4 \mathrm{~s}$ from $500-1700 \mathrm{~cm}^{-1}$ were recorded and the mean data were extracted. Prior to each step the blank spectrum was collected to quantify the absorbance.

\section{Statistical analysis}

The statistical analysis was performed using the software OriginPro 2016 (OriginLab, Northampton, USA). $t$-Tests and one-way ANOVA were used to evaluate statistical significance, followed by post hoc least significant difference tests. All data are presented as mean \pm standard deviation (SD). The error bars presented in this work are standard errors calculated using the Student's $t$-test at $95 \%$ confidence. All micrographs and images are representative of at least three independent samples imaged through three different regions of interest (ROIs). The differences were considered significant for $p$ value $<0.05$, and very significant for $p$ value $<0.01$.

\section{Conflicts of interest}

Authors state no conflict of interest.

\section{Acknowledgements}

The authors thank Gunther Richter and Hilda David for providing access to their SEM and EDAX facility and Kristen Kozielski for proof reading. A. V. S. thanks Max Planck Institute for Intelligent Systems for the grass root project grants in 2017 (M10335) and 2018 (M10338). Y. A. thanks the Alexander von Humboldt Foundation for the Humboldt Postdoctoral Research Fellowship. Open Access funding provided by the Max Planck Society.

\section{References}

1 N. Li, P. Zhao and D. Astruc, Angew. Chem., Int. Ed., 2014, 53, 1756-1789.

2 A. V. Singh, M. Batuwangala, R. Mundra, K. Mehta, S. Patke, E. Falletta, R. Patil and W. N. Gade, ACS Appl. Mater. Interfaces, 2014, 6, 14679-14689.

3 N. R. Jana, L. Gearheart and C. J. Murphy, J. Phys. Chem. B, 2001, 105, 4065-4067.

4 S. Hassan and A. V. Singh, J. Nanosci. Nanotechnol., 2014, 14, 402-414.

5 M. P. Monopoli, C. Åberg, A. Salvati and K. A. Dawson, Nat. Nanotechnol., 2012, 7, 779.

6 A. V. Singh, T. Jahnke, V. Kishore, B. W. Park, M. Batuwangala, J. Bill and M. Sitti, Acta Biomater., 2018, 71, 61-71.

7 N. Kröger, R. Deutzmann and M. Sumper, Science, 1999, 286, 1129-1132.

8 L. Chen, F. Ji, Y. Xu, L. He, Y. Mi, F. Bao, B. Sun, X. Zhang and Q. Zhang, Nano Lett., 2014, 14, 7201-7206. 
9 P. Atanasova, D. Rothenstein, J. J. Schneider, R. C. Hoffmann, S. Dilfer, S. Eiben, C. Wege, H. Jeske and J. Bill, Adv. Mater., 2011, 23, 4918-4922.

10 W. A. El-Said, H.-Y. Cho, C.-H. Yea and J.-W. Choi, Adv. Mater., 2014, 26, 910-918.

11 R. R. Kumar, S. Priya, T. S. Kumar, R. Omkumar, A. John and T. Pradeep, Langmuir, 2005, 21, 11562-11567.

12 S. Si, R. R. Bhattacharjee, A. Banerjee and T. K. Mandal, Chem. - Eur. J., 2006, 12, 1256-1265.

13 S. Negrini, V. G. Gorgoulis and T. D. Halazonetis, Nat. Rev. Mol. Cell Biol., 2010, 11, 220.

14 P. R. Sajanlal, T. S. Sreeprasad, A. K. Samal and T. Pradeep, Nano Rev., 2011, 2, 5883.

15 C. J. Murphy, T. K. Sau, A. M. Gole, C. J. Orendorff, J. Gao, L. Gou, S. E. Hunyadi and T. Li, J. Phys. Chem. B, 2005, 109, 13857-13870.

16 A. P. Middelberg, C. J. Radke and H. W. Blanch, Proc. Natl. Acad. Sci. U. S. A., 2000, 97, 5054-5059.

17 L. Gu, W. Sigle, C. T. Koch, B. Ögüt, P. A. van Aken, N. Talebi, R. Vogelgesang, J. Mu, X. Wen and J. Mao, Phys. Rev. B: Condens. Matter Mater. Phys., 2011, 83, 195433.

18 X.-B. Xu, J.-S. Luo, M. Liu, Y.-Y. Wang, Z. Yi, X.-B. Li, Y.-G. Yi and Y.-J. Tang, Phys. Chem. Chem. Phys., 2015, 17, 2641-2650.

19 G. Zhan, L. Ke, Q. Li, J. Huang, D. Hua, A.-R. Ibrahim and D. Sun, Ind. Eng. Chem. Res., 2012, 51, 15753-15762.
20 S. S. Shankar, A. Rai, B. Ankamwar, A. Singh, A. Ahmad and M. Sastry, Nat. Mater., 2004, 3, 482.

21 G. Zorn, L.-H. Liu, L. Árnadóttir, H. Wang, L. J. Gamble, D. G. Castner and M. Yan, J. Phys. Chem. C, 2014, 118, 376-383.

22 W. A. El-Said, T.-H. Kim, H. Kim and J.-W. Choi, PLoS One, 2011, 6, e15836.

23 K. C. Bantz, A. F. Meyer, N. J. Wittenberg, H. Im, Ö. Kurtuluş, S. H. Lee, N. C. Lindquist, S.-H. Oh and C. L. Haynes, Phys. Chem. Chem. Phys., 2011, 13, 11551-11567.

24 L. Wang, B. Mizaikoff and C. Kranz, J. Chem. Educ., 2009, 86, 1322.

25 A. L. Jenkins, R. A. Larsen and T. B. Williams, Spectrochim. Acta, Part A, 2005, 61, 1585-1594.

26 A. V. Singh, B. M. Bandgar, M. Kasture, B. L. V. Prasad and M. Sastry, J. Mater. Chem., 2005, 15, 5115-5121.

27 H. Hosseinkhani, P.-D. Hong and D.-S. Yu, Chem. Rev., 2013, 113, 4837-4861.

28 Y. Su, X. Wei, F. Peng, Y. Zhong, Y. Lu, S. Su, T. Xu, S.-T. Lee and Y. He, Nano Lett., 2012, 12, 1845-1850.

29 G. Von Maltzahn, J.-H. Park, A. Agrawal, N. K. Bandaru, S. K. Das, M. J. Sailor and S. N. Bhatia, Cancer Res., 2009, 69, 3892-3900.

30 Y. Wang, K. C. L. Black, H. Luehmann, W. Li, Y. Zhang, X. Cai, D. Wan, S.-Y. Liu, M. Li, P. Kim, Z.-Y. Li, L. V. Wang, Y. Liu and Y. Xia, ACS Nano, 2013, 7, 2068-2077. 